\title{
Beyond artificial intelligence
}

\section{Does a program help us to make sense of the world?}

\author{
What is Thought? \\ by Eric B. Baum \\ Bradford (MIT Press): 2004. 495 pp. \\ $\$ 40, £ 25.95$
}

\section{Igor Aleksander}

"What is thought?" is not a new question. For Aristotle, thought was what the soul does, and for Descartes it was the unequivocal evidence of one's existence. For Eric Baum, a US expert in machine learning, it is a computer program. This is not a superficial assertion: Baum pursues the idea with elegance, clarity and considerable persuasion. His choice of book title was inspired by Edwin Schrödinger's book What is Life?, which was published before the discovery of DNA. Schrödinger felt that the answer to "What is life?" must lie within physics and chemistry, despite their apparent failure to deliver this at the time. Similarly, Baum argues that an answer to "What is thought?" must lie within computer science, despite its failure to produce convincing results so far under the guise of artificial intelligence.

It is important not to treat the idea that thought is a program in too superficial a fashion. Popular texts often include explanations such as "brain is like hardware and mind is like software". Baum intends a level of sophistication far above this. The program to which he refers is one that extracts meaning from complex data. Thought for him is the process that 'understands' the complexities of the world. So a thought program is one that detects sensory information as a compact code.

For example, when walking down the road we may see a dog coming towards us. Is it friendly or not? We will need to take different actions depending on the answer. What thoughts are taking place in our heads and where do they come from? They involve a heavy dose of recalled experience and even just-learned information. We might once have been bitten by a pit-bull terrier, or just know from pictures or descriptions by others that if the dog is unmuzzled and untethered then it might be best to seek refuge behind a door, for example.

All the information we need to make our decision is there in the sensory world, but its meaning needs to be decoded from myriad other data that are not relevant to the key issue, danger in this case. There may be people, cars, other dogs, leaves carried on the wind and so on. But our thought decodes 'danger' and plans a reaction to it. Calling this thinking activity a 'decoding and planning program' requires several caveats. First, it is unlikely that a human could have written

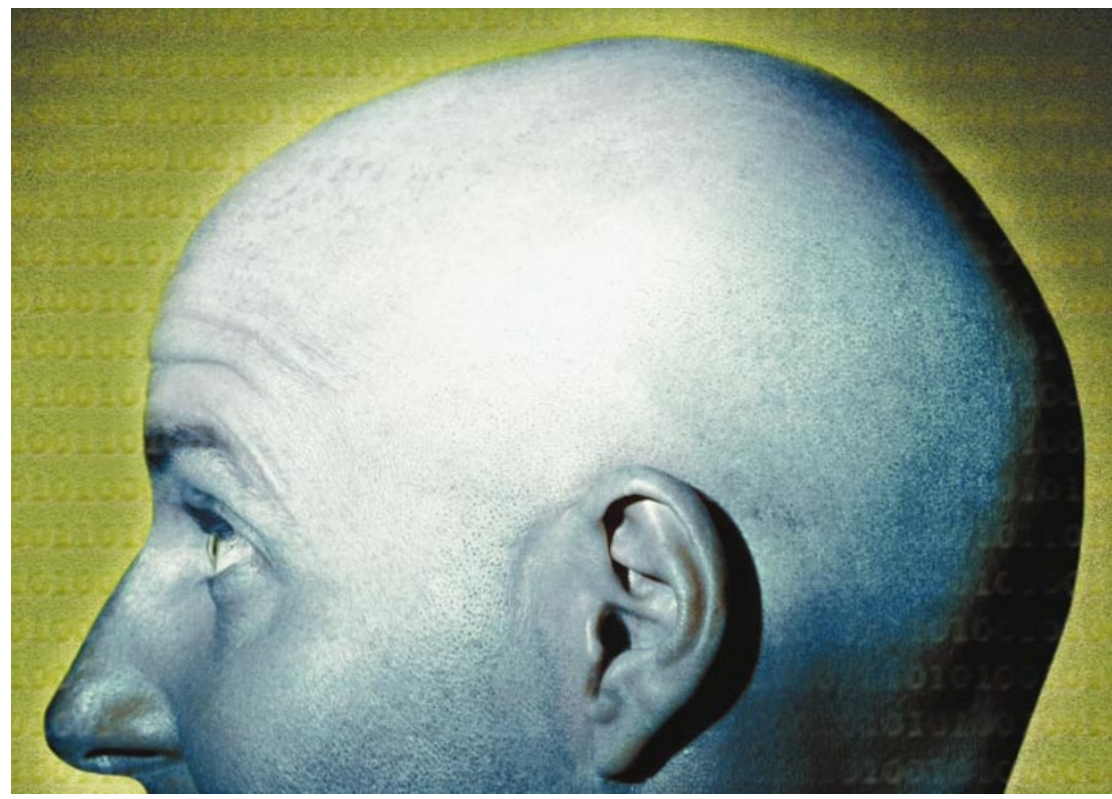

Thinking ahead: is thought a program that decodes sensory information so we can make decisions?

a program that could lead to a lifetime of thought. On the other hand, evoking an Almighty programmer is a recourse to mysticism that would leave us befuddled rather than enlightened. Baum's central point is that it is quite possible for programs to evolve, adapt and learn, making them more powerful than anything that a programmer can concoct.

A second caveat is that Baum does not suggest that someone is going to create an evolutionary, adaptive, learning program, put it into a robot and create a thinking object indistinguishable from a human being. Rather, Baum's argument is that a good (if not the best) way of understanding human thought is to analyse it as if it were a program. Artificial intelligence, in the past, was the product of programmers writing smart programs that do clever things, such as beating Garry Kasparov at chess. Baum goes beyond this by presenting clear explanations of what it is to extract the compactly coded information in the world using the simplest possible program; what it is for such a program to come into being through a process of evolution and adaptation; and what it is for a program to learn both over several generations and during daily life. Baum calls this 'Occam's razor' programming, stressing that the simplest program model is likely to have the best powers of explanation.

The third caveat is my own. One must understand that Baum's explanation is a metaphorical or functional one - it is not a material model of the brain. Even though some of his computational models are'neural networks' (a technology loosely inspired by the operation of brain cells), Baum does not suggest that a careful analysis of the brain will reveal that it is structured like a generalpurpose computer on which the meaningextraction programs run. His point is that meaning computation is a powerful explanatory metaphor for what the brain, its complex neurons, its chemical transmitters, its muscular engagement with the world and its highly adapted and impenetrable architecture do in an abstract sense. Although Baum does attempt to answer questions about what kind of program is needed for awareness, consciousness and will, he does so at a metaphorical level and does not address what the material brain does that makes it appear to a computer theoretician to be running these programs.

An important part of the book is devoted to the considerable flak that artificial intelligence scientists attracted in the heyday of this topic. Baum gives a reasoned response to John Searle's claim that no program can 'understand' the world, and to Roger Penrose's contention that conscious insight lies outside the logic that can be achieved by computation. The essence of the response is that these objections were made before ideas such as evolution, adaptation and compact decoding had become part of the idea of computation. Baum joins in the criticism of what is now called GOFAI, or 'good old-fashioned artificial intelligence', so 
this is a splendid book for discovering what is new. It will enthral some computer scientists and provoke some philosophers. And it should engage general readers who wish to enjoy a clear, understandable description of many advanced principles of computer science.

Igor Aleksander is emeritus professor and Leverhulme fellow in intelligent systems and networks at Imperial College, London SW7 2BT, UK. He is writing Five Steps to Being Conscious: the Inner Life of People, Animals and Machines.

\section{Split decision}

\section{The Fly in the Cathedral: How a Small Group of Cambridge Scientists Won the Race to \\ Split the Atom \\ by Brain Cathcart \\ Viking: 2004. 320 pp. $£ 14.99$}

\section{Frank Close}

If an atom were expanded to the size of a cathedral, its nucleus would be the size of a fly. Ernest Rutherford deduced the existence of this atomic 'fly' some 90 years ago, but the challenge of illuminating its mysteries and determining its make-up seemed to be beyond reach. Then, after years of effort, two young researchers, John Cockcroft and Ernest Walton, under Rutherford's guidance, built their eponymous accelerator in a basement at the Cavendish Laboratory in Cambridge, UK. In 1932 the media heralded their triumph with a phrase that has rung through the ages: "The atom split". The age of accelerators and big science had arrived. The Fly in the Cathedral tells the story of how this came to be.

Books about theoretical physics abound; those about experiments are thinner on the ground. The Fly in the Cathedral is indeed a rarity in focusing on how a classic experiment came about. The book's strength is that it reveals the nature of science - in particular how inspiration is partner to vast amounts of perspiration, commitment and dedication.

Rutherford discovered the existence of the atomic nucleus by scattering alphaparticles from it. No one knew how a positively charged alpha-particle managed to survive inside the atom along with all the other positive charges. But they knew that some were ejected "as if hurled by a catapult" at tremendous speed, thanks to repulsive electric forces. It was by scattering these natural bullets from atoms that Rutherford came to discover the atomic nucleus. To understand how the nucleus was constructed, it would be necessary for beams of alpha-particles to penetrate it, but first they would have to be given even higher energies to overcome the huge electrical barrier. An

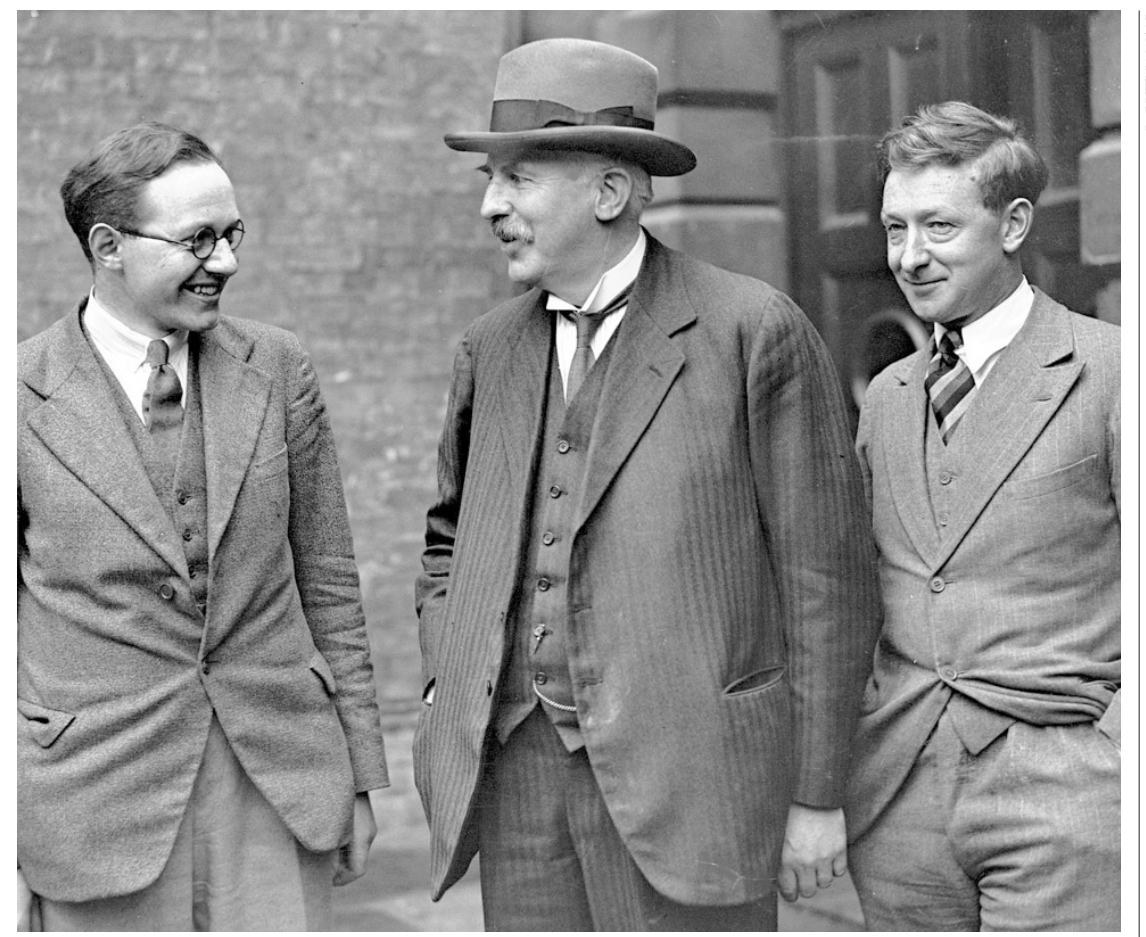

Ernest Rutherford (centre) helped Ernest Walton (left) and John Cockcroft to split the atom.

accelerator powered with millions of volts was needed. This was what Cockcroft and Walton set out to make.

Quantum mechanics came to the rescue. George Gamow showed that alpha-particles could be trapped within a huge potential barrier inside the nucleus and that, according to quantum mechanics, there was a chance that the alpha-particles could 'tunnel' through it and escape. His beautiful exposition is now a standard example in undergraduate quantum-mechanics courses. A good idea is rarely yours alone, though, and Gamow was beaten into print by Edward Condon and R. W. Gurney, even though their letter was dated one day after his. His fortune was that he came to the attention of Rutherford, which in turn led to his move to Cambridge.

Gamow gained his own priority, though, with a seminal paper in Nature in which he turned the original question on its head. Instead of discussing how particles manage to escape from the nucleus, he looked at how they might get in. Quantum mechanics enables alpha-particles to penetrate the potential Coulomb barrier without the need for them to be accelerated to huge energies. Cockcroft realized that if protons were used instead of alpha-particles, things would be even better. Instead of millions of volts, as they had originally thought, as little as 300,000 volts might be enough. This opened the way for the experimental study of nuclear physics.

In Cambridge, Cockcroft and Walton were hard at work building their device. In the United States, Ernest Lawrence was building the first cyclotron. This promised far greater energies than Cockcroft and Walton's device, but Lawrence's claims were based more on hope than realization. The pace of Brian Cathcart's narrative slows somewhat when describing the details of Cockcroft and Walton's efforts, but the slightly ponderous tone at this point is a metaphor for what actually happened: Cockcroft and Walton seemed obsessed with building and improving their machine until Rutherford one day insisted that they actually put it to work.

Suddenly the story comes alive again. Gamow's estimates, which everyone was treating with caution and aiming to beat, turned out to be conservative. Protons would invade a nucleus at relatively moderate energies easily within the reach of Cockcroft and Walton's device. It transpired that they could have achieved their results with the prototype accelerator they built some three years earlier. The rest, as they say, is history.

In addition to the history, it is unusual to see the portraits, both literal and visual, of the protagonists and appreciate their commitment to seeing the task through. For all the hyped speculation about superstrings, higher dimensions and theoretical science that borders fact and fiction, it is good to see books such as The Fly in the Cathedral, which remind us that it is ultimately experiment that decides. And that creating tools to expand our senses can provide a fascinating and dramatic story.

Frank Close is at Exeter College and the Rudolf Peierls Centre for Theoretical Physics, 1 Keble Road, Oxford University, Oxford OX1 3NP, UK. 